\title{
Nihonium the obscure
}

\section{Iulia Georgescu explains her fascination with the elusive element 113.}

C onfused by the empty boxes at the bottom-right corner of the periodic table, my friend and I bugged our high-school chemistry teacher until she produced a newer version from a back cabinet. There, those boxes were filled with obscure element symbols such as Uut, Uuq or Uup, associated with nearunpronounceable latinized placeholder names that simply stood in for the atomic numbers: Uut for ununtrium, which just means 1-1-3-ium, Uuq for ununquadium, or 1-1-4-ium, and so on. None of this did anything to help illuminate us. We were then sent away with a brief mention that these were short-lived man-made elements created in a nuclear reactor. This notentirely-accurate explanation was rather unsatisfying. Undeterred, we went straight to the bookshop, purchased a periodic table poster, stuck it on the wall of my room and promptly proceeded to fill in the empty boxes with new elements shamelessly named after our ourselves, our surnames germanized for good measure.

My career did not live up to my exalted teenage expectations and unsurprisingly there is no element named after me, but years later I was working as a postdoc at RIKEN (Rikagaku Kenkyūsho, or the Institute of Physical and Chemical Research) in Japan and met Kosuke Morita, who led the team that discovered element 113 - the Uut that had intrigued me back in school. For a long time I had no idea that the jovial, unassuming man that I was regularly meeting at the Go club playing the popular strategy board game was actually a well-known superheavy element hunter. But this is not the only reason I have a special affinity for element 113. I associate it with the exotic and sometimes misunderstood culture of its eponymous country.

Element 113 - named nihonium (Nh) after Nihon, which means Japan in Japanese - was the first element discovered and correctly identified in Asia. We need "and correctly identified" because of its

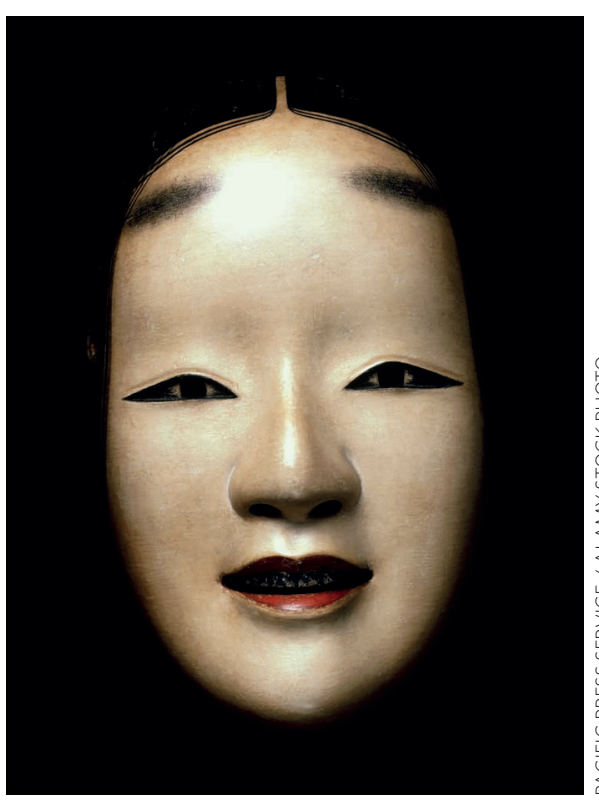

problematic cousin nipponium (named after Nippon, synonym of Nihon). At the beginning of the twentieth century the Japanese chemist Masataka Ogawa was studying with William Ramsay in London. They suspected some new element could be found in a mineral called thorianite. Ogawa managed to isolate what was indeed a new element and published his results in 1909, naming it nipponium - he had misidentified it as element 43 (now technetium), however, when it was in fact element 75 (rhenium), right below it in the periodic table. Element 43 was mistakenly claimed for another time by German chemists Walter and Ida Noddack and Otto Berg, who all actually went on to discover rhenium in 1925 - completely independently from Ogawa's unrecognized element. Finally, element 43 was discovered - and correctly identified - by Emilio Segrè and Carlo Perrier in 1937.

Nihonium's discovery was perhaps less dramatic, but element 113 was arguably far more difficult to spot and identify. In 2003 Morita's team started looking for superheavy elements at RIKEN's heavyion linear accelerator. Just a year later they had already managed to synthesize the first nihonium atom by smashing bismuth-209 and zinc-70 nuclei. The atom decayed after a mere 0.34 milliseconds and the challenge was to identify it through a forensic analysis of its decay products. Nihonium decays sequentially to dubnium-262 through four $\alpha$-decays, which in turn further breaks down into two smaller fragments. In 2005 the RIKEN team managed to identify the remains of another nihonium atom.

To fully characterize the dubnium decay chain the RIKEN team still had two $\alpha$-decays to understand, from bohrium-266 to dubnium-262 and lawrencium-258. For this, they bombarded curium with a sodium beam to obtain bohrium and dubnium, and once this process was clear they then 'just' had to wait for another nihonium atom to be generated. It took seven years and a lot of patience, but in 2012 Morita's team finally identified a chain of six $\alpha$-decays from element 113, this time not to dubnium but further down to lawrencium-258 and mendelevium-254. The existence of nihonium was finally nailed down.

Meanwhile, a Russian-US collaboration between the Dubna Joint Institute for Nuclear Research and the Lawrence Livermore National Laboratory had also identified element 113 as a decay product of element 115 , which they would name moscovium, and made a discovery claim for these two elements. By 2005 the collaboration had strengthened their case for the observed decay, but the IUPAC still found the evidence insufficient and the discovery of element 113 was attributed to the RIKEN team, who were given the privilege of naming it.

Although it's now been produced, identified and named, we still haven't learned an awful lot more about element 113 since my school years. Nihonium makes me think of a Japanese Noh mask (pictured): mysterious and somewhat disturbing. We don't know what is hiding behind it; for example, in terms of its properties or behaviour, but perhaps that is where its charm lies.

IULIA GEORGESCU is the Chief Editor of Nature Reviews Physics. 